\title{
ESTIMACIÓN DE LOS PERIODOS NATURALES DE VIBRACIÓN DE VIVIENDAS DE BAJA ALTURA CON MUROS DE CONCRETO
}

\section{ESTIMATION OF THE NATURAL VIBRATION PERIODS IN TRADITIONAL HOUSING WITH CONCRETE WALLS}

Wilmer Julián, Carrillo León

Ing. Civil, MSc. Profesor asistente, Facultad de Ingeniería, Universidad Militar Nueva Granada, Bogotá, Colombia. Candidato a Doctor en Ingeniería, Universidad Nacional Autónoma de México, wcarrillo@umng.edu.co

Fecha de recepción: 27 de enero de 2009

Fecha de aprobación: 11 de junio de 2009

\section{RESUMEN}

En el artículo se presentan los periodos de vibración de viviendas típicas con muros de concreto, con el propósito de evaluar dos técnicas de modelación que se utilizan en la práctica del diseño estructural. Los resultados analíticos se compararon con los obtenidos a partir de pruebas experimentales de vibración ambiental. En el programa de investigación se incluyeron edificaciones de uno y dos niveles con configuraciones típicas de viviendas de baja altura en México. Aunque existen incertidumbres significativas en la creación de modelos de análisis, se considera que las dos metodologías numéricas son apropiadas para la estimación de los periodos de vibración de este tipo de estructuras. Tal como se esperaba, los resultados del método de elementos finitos son más cercanos a los experimentales. Adicionalmente, los periodos de vibración obtenidos con el método de la columna ancha son mayores que los periodos obtenidos con el método de elementos finitos.

Palabras claves: periodo de vibración, muros de concreto, prueba de vibración ambiental, modelos numéricos, método de la columna ancha, elementos finitos.

\section{ABSTRACT}

This research is to evaluate two modeling techniques used in the practice of structural design by the vibration periods in traditional housings with concrete walls. The analytical results were compared with the results obtained by atmosphere vibration testings. In the research was taken one and two floor housing with traditional low walls in Mexico. Although there are significant uncertainties to carry out analytical models, it is conside- 
red that the two analytical methodologies are suitable for estimating vibration periods of this type of structures. Just as it was expected, the results of the finite element method are closer to the experimental findings. Also, vibration periods achieved with the wide column method are higher than the experimental ones.

Key words: vibration period, concrete walls, ambient vibration test, numeric models, wide column method, finite elements.

\section{INTRODUCCIÓN}

La estimación del periodo natural de vibración de una estructura de concreto reforzado es un procedimiento esencial en el diseño sísmico. A partir de esta característica puede obtenerse una buena apreciación de las demandas globales sobre una estructura sometida a una determinada acción sísmica. Esta propiedad depende de la masa y la rigidez de la estructura, y es afectada por muchos factores tales como la regularidad de la estructura, el número de pisos y claros, las dimensiones de las secciones, el nivel de carga axial, las cuantías de refuerzo y del nivel de agrietamiento del concreto.

Para determinar los periodos de vibración de viviendas de muros de concreto se utilizan comúnmente ecuaciones simplificadas (Chopra y Goel, 2000; Chun et al., 2000; Goel y Chopra, 1998; Aristizabal-Ochoa, 1983; entre otras), o en el mejor de los casos se construyen modelos numéricos en los cuales se consideran ciertas hipótesis en algunos aspectos estructurales. Sin embargo, existen grandes incertidumbres del comportamiento real ante excitaciones dinámicas, entre los que se destacan los efectos de interacción suelo-estructura, los efectos de torsión, la flexibilidad del diafragma de piso, la efectividad de las juntas constructivas y la participación de elementos no estructurales. El considerar en forma errónea la participación de estos aspectos estructurales puede conducir a estimaciones poco confiables de los periodos de vibración y amortiguamientos naturales de las edificaciones, parámetros importantes que gobiernan la respuesta dinámica de las mismas (Muriá et al., 2000; Muriá y González, 1995).

Con el propósito de estudiar el comportamiento de los muros de concreto de baja altura ante acciones sísmicas, se desarrolló un extenso programa de investigación entre el Instituto de Ingeniería de la UNAM y el Grupo CEMEX de México (Carrillo y Alcocer, 2008, 2008a). Como parte de dicho proyecto se estudió la efectividad de algunas técnicas de modelación numérica como herramientas para calcular los periodos de vibración de viviendas de baja altura a base de muros de concreto. Para ello se llevaron a cabo pruebas experimentales de vibración ambiental y se realizaron modelos numéricos en varias configuraciones típicas de vivienda (Carrillo, 2009). A partir de los resultados obtenidos, en el artículo se establecen las limitaciones y bondades de las técnicas de modelación numérica utilizadas, y se discuten algunos de los parámetros recomendados en los reglamentos para el diseño de este tipo de estructuras. 


\section{TÉCNICAS DE IDENTIFICACIÓN}

Para estimar los periodos de vibración de estructuras es posible utilizar dos técnicas de identificación: (a) técnicas no paramétricas que consisten en determinar las características del sistema estructural con base en análisis espectrales convencionales de pruebas de vibración ambiental y, (b) técnicas paramétricas donde se establece un modelo numérico simplificado y se estiman los valores de los parámetros estructurales necesarios para producir una buena correlación entre la respuesta medida y la calculada.

\subsection{TÉCNICAS PARAMÉTRICAS: MODELACIÓN NUMÉRICA}

Para el intervalo del comportamiento elástico lineal, las deformaciones de un muro ante cierto caso de cargas en su plano, deben calcularse con los métodos y teorías de la elasticidad. Para esto, es necesario tener en cuenta las propiedades elásticas del material, la magnitud y distribución de las cargas, la geometría del muro y la forma en que esté apoyado. Existen diferentes soluciones dependiendo del nivel de complejidad de la geometría o las condiciones de frontera.

El método de la columna ancha (MCA), es uno de las soluciones más aproximadas para el análisis de estructuras a base de muros. Consiste en concentrar las propiedades de los muros (o segmentos de muro) en columnas ficticias ubicadas en el centro de los mismos (eje), las cuales tendrán las características geométricas del muro que representan (figura 1a).

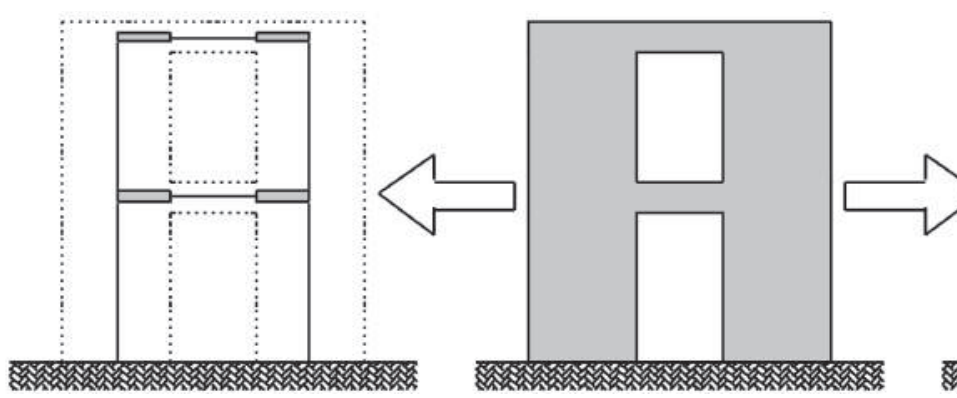

(a) MCA

Figura 1. Aplicación de métodos numéricos.

El método tiene la ventaja de que este tipo de sistemas se idealizan como estructuras esqueletales, lo mismo que los pórticos (Bazán y Meli, 1992; Flores y Alcocer, 2001). Hoy en día, el método del elemento finito (MEF), constituye una de las más poderosas herramientas para el análisis de estructuras complejas, como ciertos muros de composición y geometría complicada (Bazán E. y Meli R., 2004). El MEF constituye un método numérico destinado a resolver, mediante ecuaciones matriciales, las ecuaciones diferenciales que se plantean en sistemas discretos (estructuras) o continuos (campos). En 
programas de computador se toma la geometría planteada y se convierte en un modelo con cierta cantidad definida de elementos (figura 1b).

\subsection{TÉCNICA NO PARAMÉTRICA: PRUEBAS DE VIBRACIÓN AMBIENTAL}

Actualmente existen tres tipos de pruebas experimentales para determinar las características dinámicas de las estructuras en escala real. Una de ellas basada en vibraciones del tránsito de vehículos, del empuje del viento y del uso de la estructura, denominada prueba de vibración ambiental (PVA). Otra metodología son las pruebas de vibración forzada (PVF), en la cual la estructura es excitada por una vibración constante con uno o más motores de vibración que tienen un control de velocidad preciso. Otro método, denominado prueba con registros sísmicos (PRS), consiste en registrar por medio de acelerógrafos los movimientos provocados por los sismos.

La principal diferencia de este tipo de pruebas es la amplitud del movimiento utilizada para determinar las características dinámicas de la estructura. Por ejemplo, las PRS tienen la ventaja de poder estudiar el comportamiento dinámico de estructuras cuando éstas se someten a amplitudes de excitación mucho mayor que las que se presentan con ruido ambiental (Muriá y González, 1995).

Adicionalmente, debido a las características de los equipos, el número de instrumentos y el tiempo que se requiere para realizar estos tipos de pruebas, la inversión económica en cada una de ellas difiere apreciablemente. Por ejemplo, realizar una PVF puede requerir mayor inversión económica que la necesaria para efectuar una PVA (Trifunac, 1972). Debido a que no se conoce la fecha de ocurrencia de un movimiento sísmico, podría ser necesario ubicar por un tiempo prolongado los instrumentos requeridos para realizar una PRS, por lo cual, su costo puede ser uno de los más elevados.

En las pruebas de vibración ambiental (PVA), a través de acelerómetros de alta resolución, se registran las vibraciones producidas en las estructuras debido a condiciones ambientales, por lo que es un método simple y rápido en la obtención de datos, con la ventaja de no interferir en las actividades normales que se realizan en los inmuebles. Las señales captadas por los acelerómetros son transmitidas a unos acondicionadores donde se amplifican y se filtran con el fin de obtener registros claros y adecuados. Empleando diferentes arreglos de acelerómetros que se localizan en puntos de medición seleccionados, es posible determinar las principales propiedades dinámicas de una estructura, entre ellas, sus periodos de vibración.

\subsubsection{Equipo utilizado}

Para este estudio se utilizó un sistema de adquisición de datos que permite capturar simultáneamente las vibraciones en ocho puntos de la estructura. En cada punto de medición elegido se coloca un servo-acelerómetro de alta resolución para detectar y 
adquirir las señales de ruido ambiental, cuyas amplitudes son entre 10-4 a 10-5 g, en un tiempo de captura de aproximadamente 10 minutos. Estas señales son trasmitidas, a través de cables blindados, a unos acondicionadores donde se amplifican y se filtran las frecuencias mayores que $30 \mathrm{~Hz}$. Las señales acondicionadas se envían a una microcomputadora en donde se almacena toda la información. Se cuenta con un analizador de espectros de dos canales, con el cual se monitorea la evolución de los espectros durante la prueba (Figura 2).

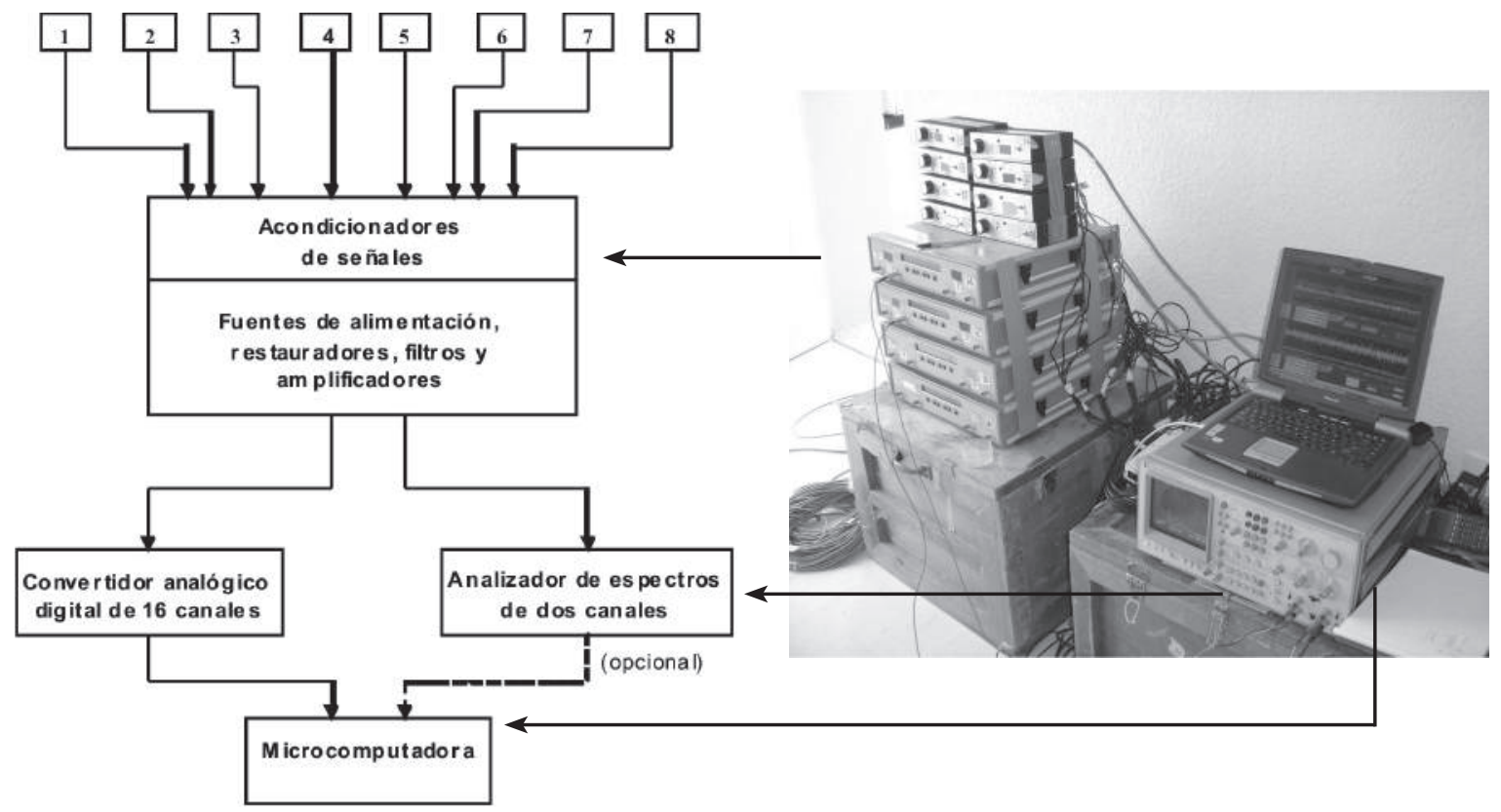

Figura 2. Diagrama de flujo del dispositivo experimental.

\subsubsection{Interpretación de resultados}

El procesamiento de la información consistió en un análisis de señales aleatorias estacionarias para obtener los espectros de densidad espectral, así como las correspondientes funciones de transferencia (en fase y amplitud) y de coherencia entre pares de señales. Para identificar las frecuencias naturales de vibración se utilizó tanto el espectro de potencia promedio y la función de transferencia como la de coherencia. De lo contrario, podrían cometerse errores si se utilizan exclusivamente los espectros de Fourier, debido a la presencia de otras ordenadas espectrales que pueden ser significativas pero ajenas a las frecuencias naturales de la edificación como son, por ejemplo, las ordenadas asociadas a vibraciones de estructuras vecinas y de equipo electromecánico (Muriá, 1997). Para identificar las frecuencias traslacionales de vibración fue necesario utilizar los espectros de potencia promedio. Lo anterior debido a que las edificaciones analizadas son de muy bajo periodo (alta rigidez y baja altura) y están 
desplantadas sobre suelo firme, lo cual genera un movimiento de cuerpo rígido que dificulta la identificación de las frecuencias propias de la estructura a partir de la función de transferencia. Sin embargo, para identificar la frecuencia de vibración rotacional se utilizó la función de transferencia en fase y amplitud, ya que las señales son adquiridas en puntos que hacen parte de la estructura.

\section{VIVIENDAS ANALIZADAS}

Para realizar la comparación de las técnicas de identificación se realizaron modelos numéricos y pruebas de vibración ambiental en diferentes configuraciones de viviendas de baja altura con muros de concreto. La modelación numérica se realizó en estructuras de uno y dos niveles. Debido a inconvenientes logísticos (ocupación de las viviendas por los usuarios), sólo fue posible realizar pruebas de vibración ambiental en tres viviendas de un nivel. A partir de los resultados experimentales en las viviendas de un nivel, se calibraron los modelos numéricos de las viviendas de uno y dos niveles.

\subsection{VIVIENDAS DE UN NIVEL}

\subsubsection{Descripción general vivienda tipo I}

Corresponde a una vivienda de interés social con muros de $10 \mathrm{~cm}$ de espesor, resistencia nominal a la compresión del concreto, fc' = $14.7 \mathrm{MPa}(150 \mathrm{kgf} / \mathrm{cm} 2)$ y módulo de elasticidad de $9600 \mathrm{MPa}(98000 \mathrm{kgf} / \mathrm{cm} 2)$. Las dimensiones globales de la vivienda entre ejes son de $5.8 \mathrm{~m}$ x $10.4 \mathrm{~m}$ (Figura 3), y tienen un área aproximada de $50 \mathrm{~m} 2$. En la losa de azotea sobresale una estructura que sirve para el almacenamiento de un tanque de agua con capacidad máxima de $1 \mathrm{~m} 3$. La configuración de estas viviendas se muestra en la Figura 4. La cimentación está conformada por vigas en las dos direcciones de $40 \mathrm{~cm}$ de base y de altura, y una losa de concreto de $10 \mathrm{~cm}$ de espesor. La cimentación fue calculada para una capacidad portante del terreno de $0.15 \mathrm{MPa}$ (15 $\mathrm{tf} / \mathrm{m} 2)$. La altura libre de los muros es de $2.4 \mathrm{~m}$.

\subsubsection{Descripción general vivienda tipo 2}

Corresponde a dos prototipos de viviendas construidas en el Centro de Tecnología del Cemento y del Concreto (CTCC) de CEMEX en México. Las dos viviendas tienen un área aproximada de $36 \mathrm{~m} 2$ (ver Figura 5), muros con espesor de $10 \mathrm{~cm}$ y altura libre de $2.4 \mathrm{~m}$. Las viviendas fueron construidas con concreto de resistencia nominal a la compresión de 14.7 MPa (150 kgf/cm2), una de ellas con concreto normal y la otra con concreto celular (ver Figura 6). La losa de la azotea se divide en dos tipos: la mitad de la vivienda tiene una losa maciza de $10 \mathrm{~cm}$ de espesor y la otra mitad, una losa aligerada en una dirección con viguetas separadas cada $60 \mathrm{~cm}$, casetón de poliestireno de $10 \mathrm{~cm}$ y losa de compresión de $5 \mathrm{~cm}$. 


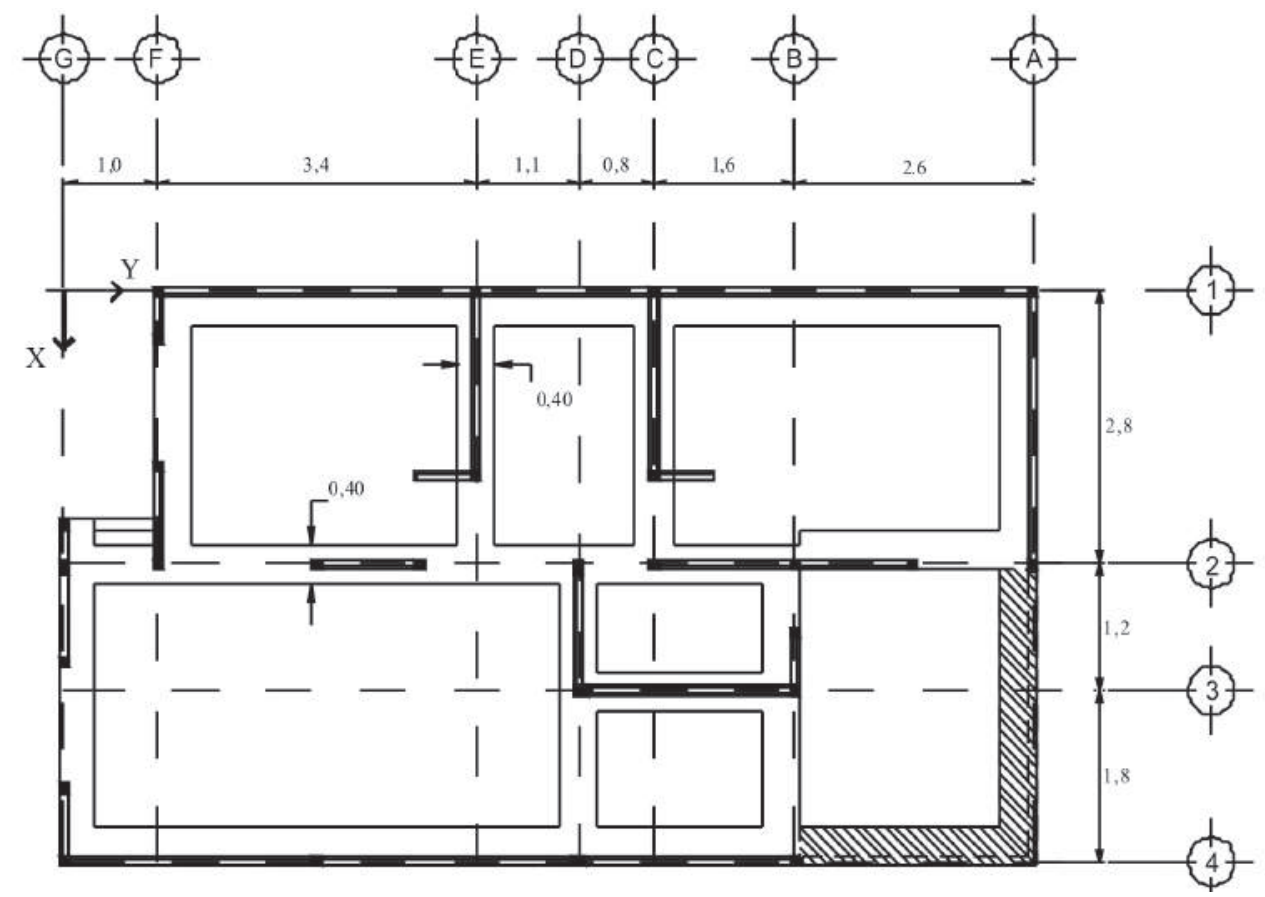

Figura 3. Planta de cimentación y de muros de la vivienda Tipo 1 de un nivel (acotaciones en m)
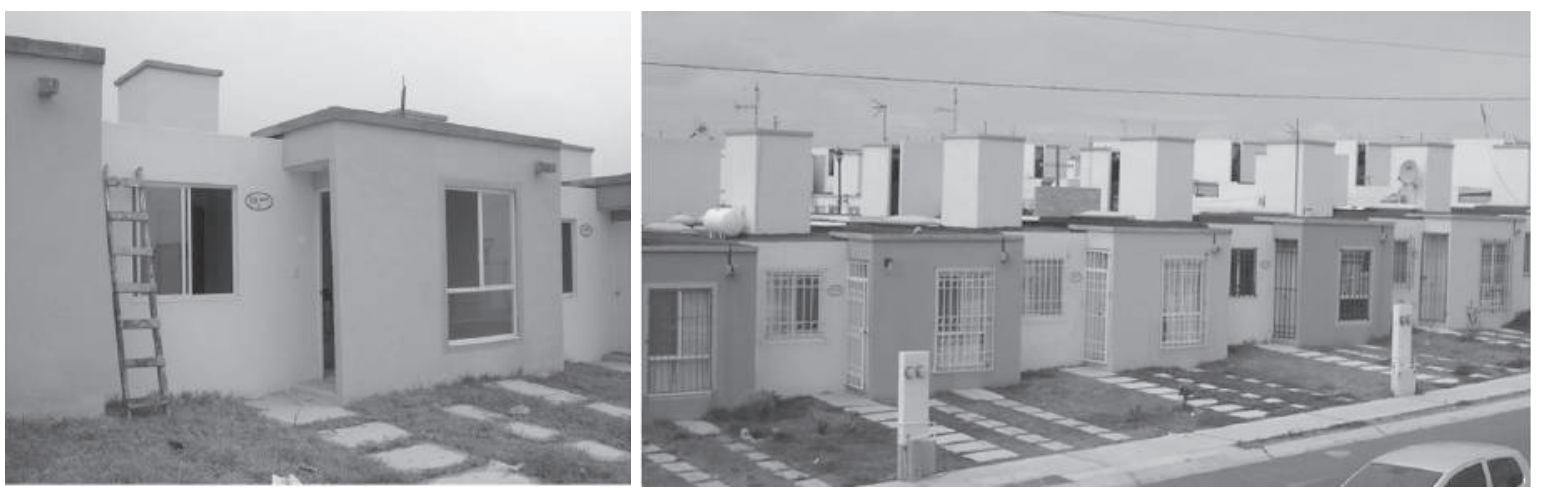

Figura 4. Configuración de las viviendas Tipo 1 de un nivel.

\subsubsection{Parámetros de modelación}

Para realizar la modelación elástica con el MCA y el MEF, se utilizó el Programa SAP2000 V10.01 (Computer and Structures, 2005). Para la evaluación de cargas (y masas) se siguieron las recomendaciones estipuladas en las Normas Técnicas Complementarias sobre Criterios y Acciones (NTC-Cr, 2004) y para diseño por Sismo (NTC-S, 2004) del Distrito Federal de México. Debido a que las viviendas se encuentran actualmente en uso, en la modelación se tuvo en cuenta la presencia de regiones fisuradas por medio de la modificación de las propiedades de los elementos, recurriendo a la recomenda- 
ción estipulada en la sección 1.4.1 de las NTC-C (2004), es decir, utilizando el 50\% de la inercia gruesa de las secciones $(0.5 \mathrm{lg})$. Las losas de entrepiso se modelaron por medio de diafragmas rígidos, donde se ubicaron los centros de masa y se aplicaron las masas traslacionales y rotacionales correspondientes.

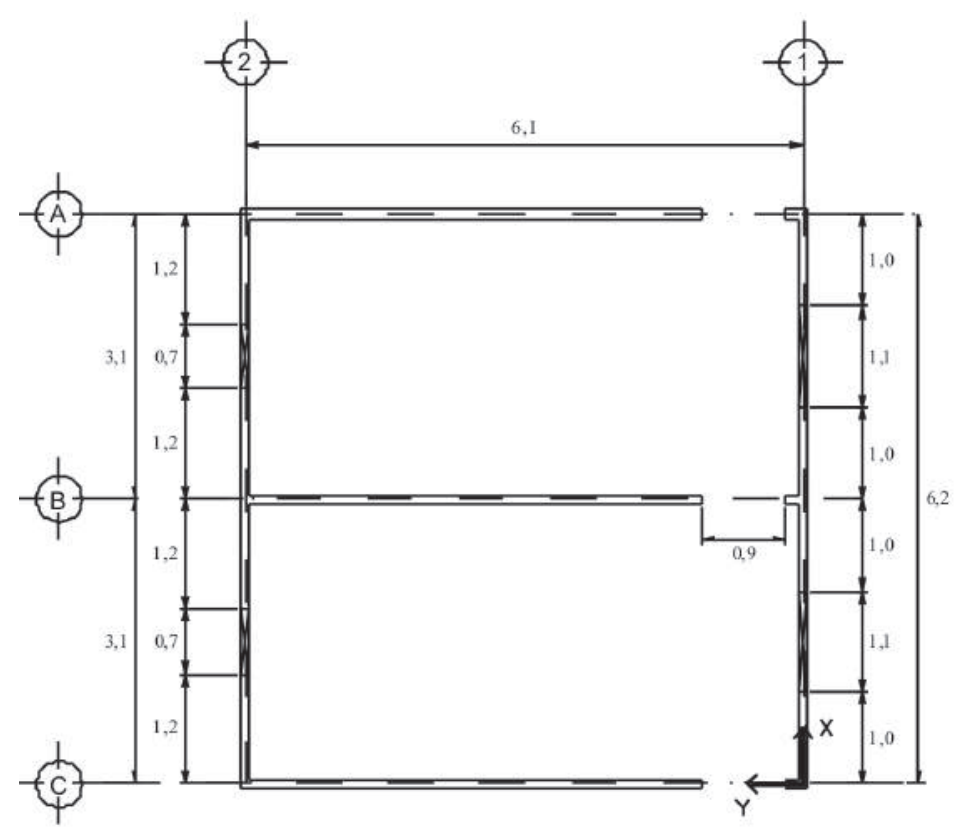

Figura 5. Planta de muros de las viviendas Tipo 2 de un nivel (acotaciones en $\mathrm{m}$ )

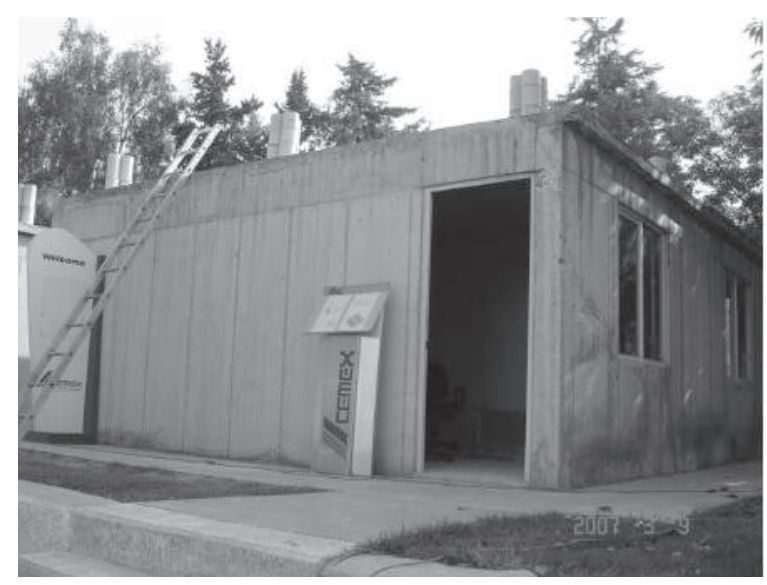

(a) En concreto normal

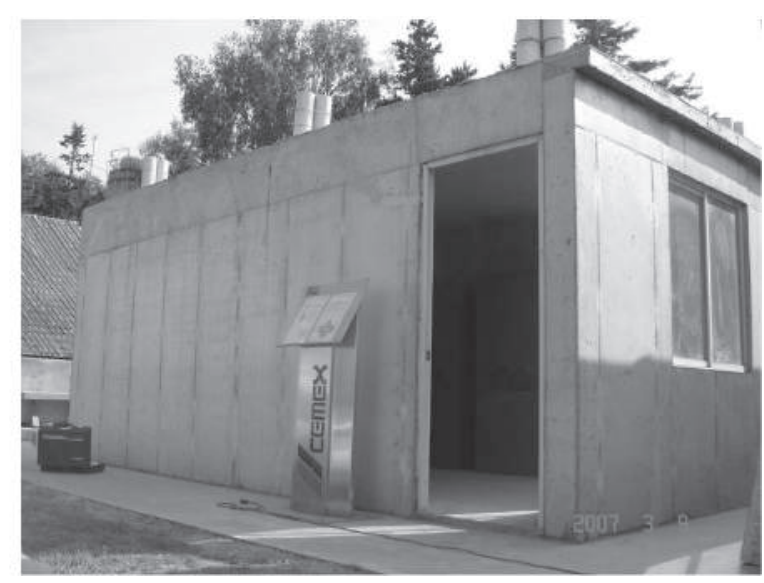

(b) En concreto celular

Figura 6. Configuración de las viviendas Tipo 2 de un nivel

Para tener en cuenta el efecto del tipo de suelo y de la cimentación de las viviendas, se modeló la interacción suelo-estructura utilizando rigideces equivalentes por medio del 
módulo de reacción del suelo (Bazán y Meli, 2004). Para esto, fue necesario modelar la losa de cimentación por medio de elementos finitos conectados por un diafragma rígido. Debido a que no se realizó un estudio geotécnico para determinar el módulo de reacción del suelo, se utilizaron valores promedio disponibles en la literatura en función del tipo y la clasificación del suelo. Para la vivienda Tipo 1 se supuso un módulo de reacción vertical del suelo, Ksv, igual a 50000 kN/m3 (5100 tf/m3). Para las viviendas Tipo 2 se supuso un módulo igual a $40000 \mathrm{kN} / \mathrm{m} 3$ (4100 tf/m3). En la dirección horizontal, para los dos tipos de viviendas, se utilizó el 30\% del módulo en la dirección vertical. A partir del área aferente a cada uno de los nodos de la losa de cimentación, se asignaron las rigideces equivalentes en las tres direcciones por medio de elementos tipo resorte.

Vivienda Tipo 1. En la modelación se utilizaron las características mecánicas nominales del concreto. Dichas propiedades se encuentran en el intervalo de medición obtenido a partir de pruebas no destructivas (equipo de esclerómetro y ultrasonido) realizadas a los muros durante la visita de campo (IMCYC, 2007). La mayoría de estas viviendas son construidas una al lado de otra, separadas por medio de una junta conformada por una lámina de poliestireno. Sin embargo, dicha junta no funciona adecuadamente ya que durante la construcción, se obstruye parcialmente con residuos de concreto. Para tener en cuenta el efecto de la junta en la modelación, se asignaron rigideces equivalentes (aproximadas) a elementos tipo resorte ubicados en los dos costados de la vivienda donde se habilita la junta. Los modelos se muestran en la Figura 7.

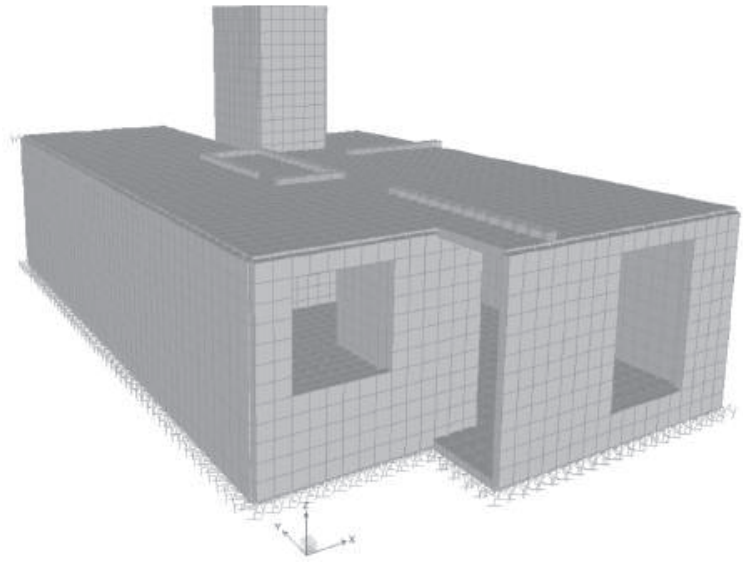

(a) MEF

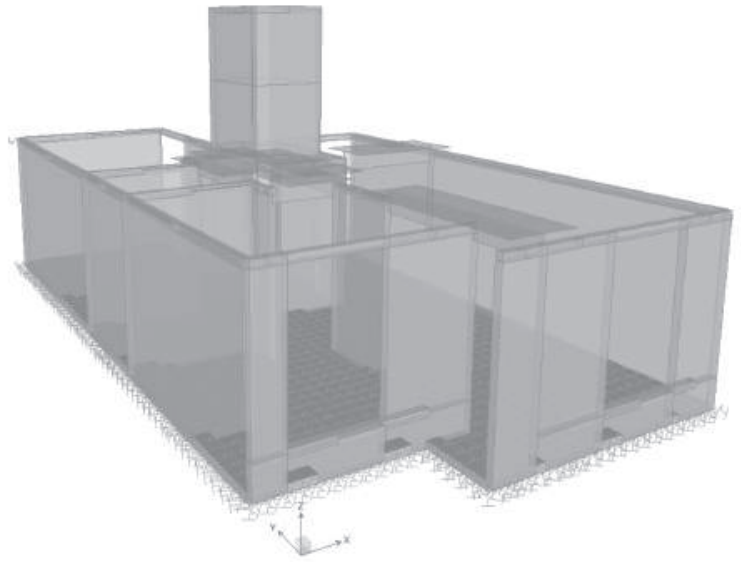

(b) MCA

Figura 7. Geometría general de los modelos de análisis de la vivienda Tipo 1 de un nivel

Viviendas Tipo 2. Las propiedades mecánicas del concreto utilizadas en la modelación fueron obtenidas experimentalmente a partir del ensaye de núcleos extraídos a los muros. Para la vivienda de concreto normal la resistencia a la compresión fue de 19.5 MPa (200 kgf/cm2) y un módulo de elasticidad de 12250 MPa (125000 kgf/cm2). Para 
la vivienda de concreto celular, estos valores fueron de $44.6 \mathrm{MPa}(455 \mathrm{kgf} / \mathrm{cm} 2)$ y de $20483 \mathrm{MPa}$ (209000 kgf/cm2). Los modelos de análisis se muestran en la Figura 8.
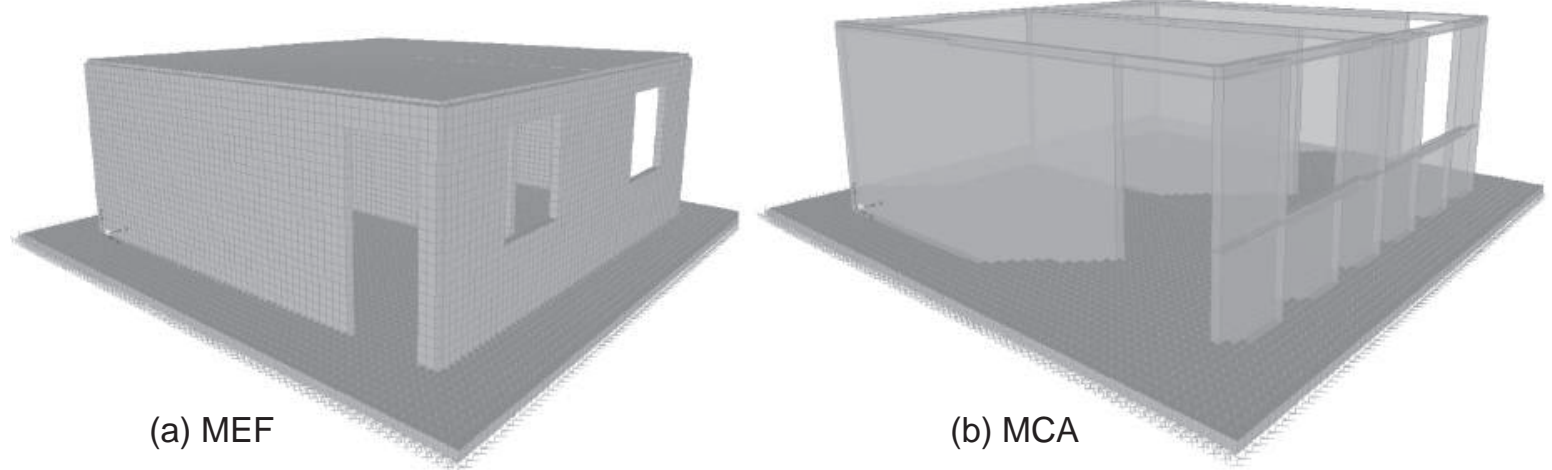

Figura 8. Geometría general de los modelos de análisis de las viviendas Tipo 2 de un nivel

\subsubsection{Pruebas de vibración ambiental}

Como se mencionó anteriormente, sólo fue posible realizar pruebas de vibración ambiental en las viviendas de un nivel. Sin embargo, los resultados obtenidos fueron esenciales para la calibración de los modelos numéricos tanto de las viviendas de uno, como de dos niveles.

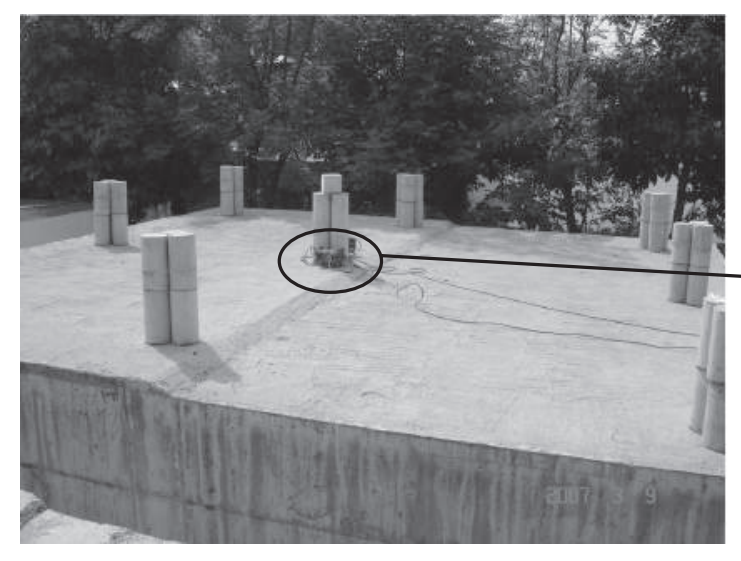

(a) Vista general

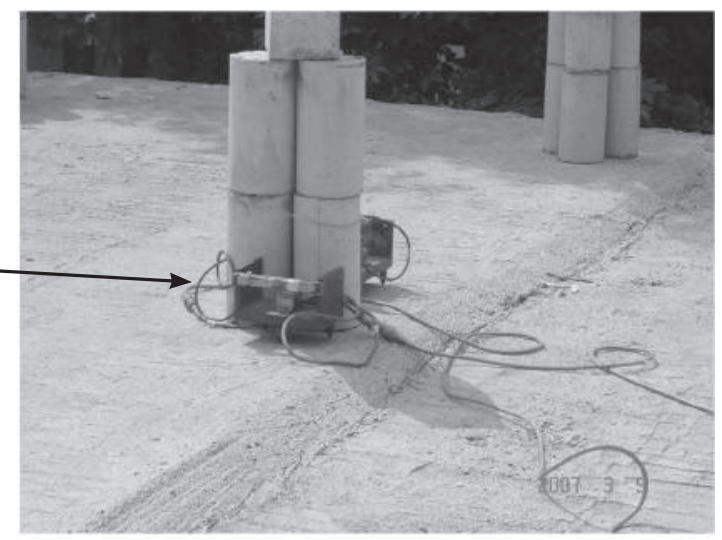

(b) Detalle

Figura 9. Ubicación en acelerómetros en la azotea de una vivienda

Descripción general de las pruebas. Las pruebas consistieron en colocar y orientar los acelerómetros cerca de los centros geométricos de los dos niveles de la estructura en sus direcciones ortogonales, las cuales se identificaron como T y L (Transversal y Longitudinal). La dirección $T$ corresponde a la dirección del eje $X$ y la dirección $L$ a la dirección del eje Y, de la Figura 3 (vivienda Tipo 1) y de la Figura 5 (vivienda Tipo 2). Se ubicaron 
dos acelerómetros en la azotea $(A C)$, dos en la planta del primer nivel y otros dos en un punto en el terreno (CL). Posteriormente, los acelerómetros de la azotea se movieron a las esquinas opuestas para determinar el modo de vibración de torsión (ver Figura 9).

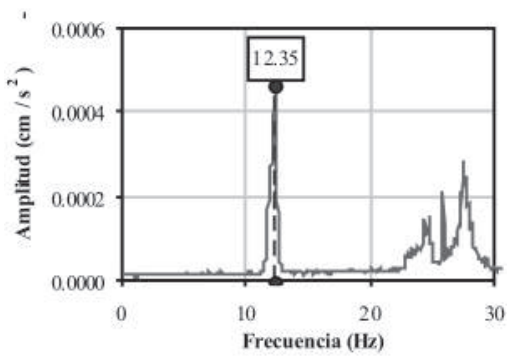

(a) EPP, $A C(T)$

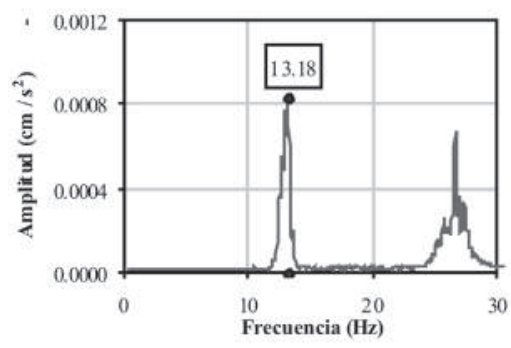

(b) EPP, $A C(L)$

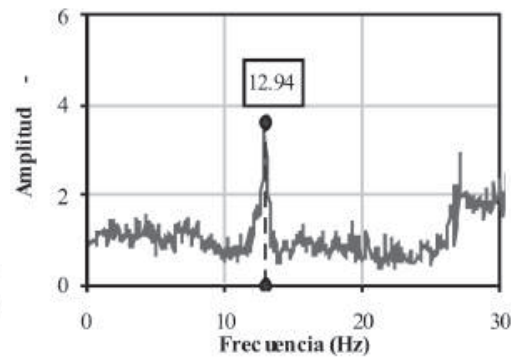

(c) FT, AC(T) / AC(L)

Figura 10. Resultados de la prueba en la vivienda Tipo 1

Procesamiento de la información. En la Figura 10 se presentan los espectros de potencia promedio (EPP) para identificar las frecuencias naturales de vibración en la dirección transversal y longitudinal, y la función de transferencia (FT) para establecer la frecuencia asociada al modo de vibración de torsión en la vivienda. En los recuadros de cada gráfica se presenta la frecuencia de vibración. En la Figura 11 y en la Figura 12 se presentan los resultados para la vivienda construida en concreto normal y la de concreto celular, respectivamente.

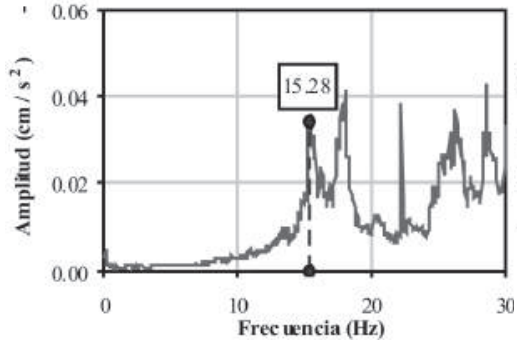

(a) EPP, AC(T)

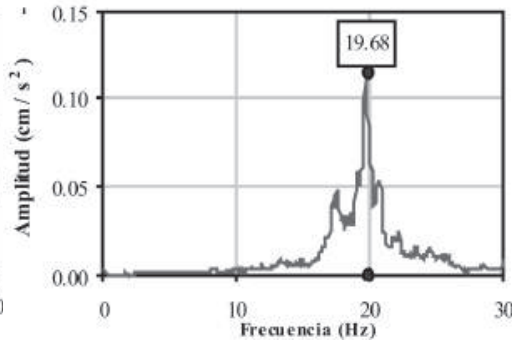

(b) EPP, AC(L)

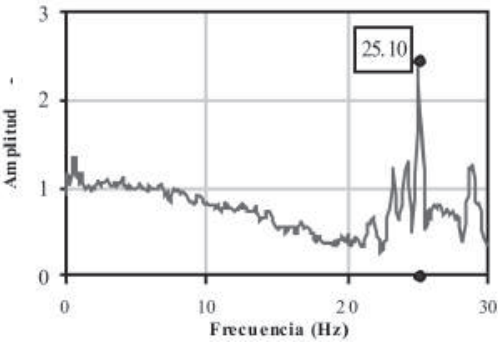

(c) FT, AC(T) / AC(L)

Figura 11. Resultados de la prueba en la vivienda Tipo 2 de concreto normal

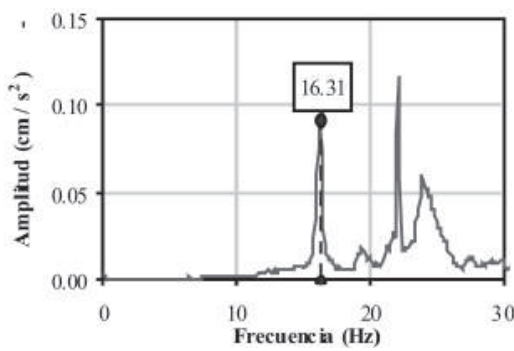

(a) EPP, AC(T)

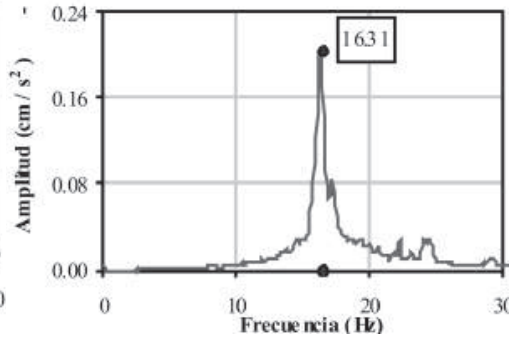

(b) EPP, AC(L)

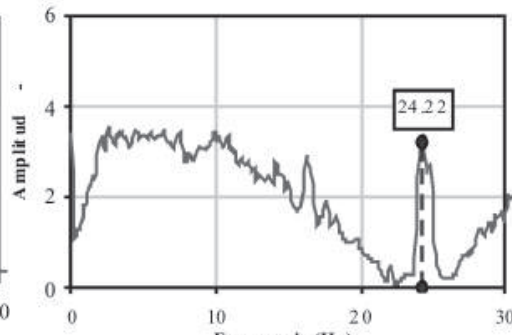

(c) $\mathrm{FT}, \mathrm{AC}(\mathrm{T}) / \mathrm{AC}(\mathrm{L})$

Figura 12. Resultados de la prueba en la vivienda Tipo 2 de concreto celular. 


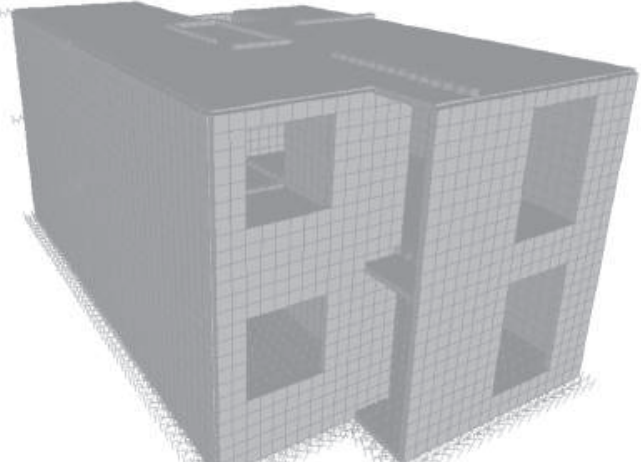

(a) MEF

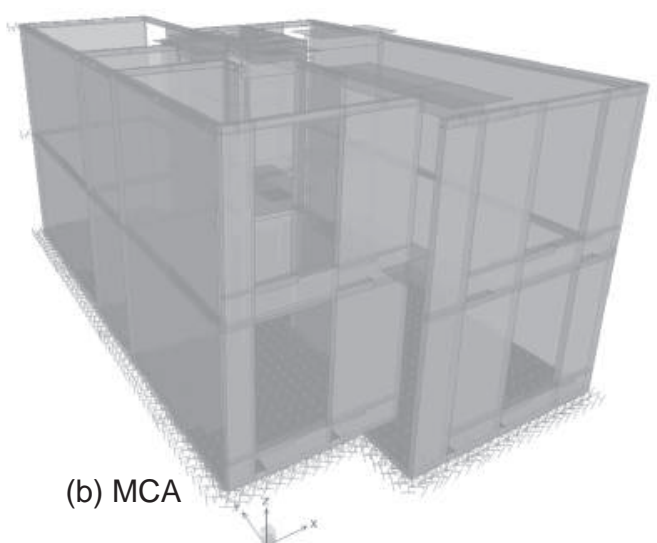

(b) MCA

Figura 13. Geometría general de los modelos de análisis de la vivienda de dos niveles.

\subsection{VIVIENDAS DE DOS NIVELES}

Corresponden a viviendas Tipo 1 de un nivel, en las cuales se prolonga la construcción hasta el segundo nivel. Por lo tanto, las características generales, la evaluación de cargas (y masas) y los demás parámetros de modelación (interacción suelo-estructura, junta entre las viviendas, etc.) son similares a las de las viviendas de un nivel. En la Figura 13 se muestran los modelos numéricos.

\section{RESULTADOS Y DISCUSIÓN}

VIVIENDAS DE UN NIVEL. En la Tabla 1 se presentan los periodos de vibración obtenidos a partir de la modelación numérica y de las pruebas de vibración ambiental de la vivienda Tipo 1. En la Tabla 2 y en la Tabla 3 se presentan los resultados para las viviendas Tipo 2 de concreto normal y celular, respectivamente. En estas tablas se presenta la relación entre los periodos analíticos y experimentales (TMEF/TPVA y TMCA/TPVA), al igual que la relación entre los periodos calculados con las metodologías numéricas (TMEF/TMCA).

Tabla 1. Periodos de vibración en la vivienda Tipo 1.

\begin{tabular}{|c|c|c|c|c|c|c|}
\hline \multirow{2}{*}{ Modo ${ }^{1}$} & \multicolumn{3}{|c|}{$\mathrm{T}(\mathrm{s})$} & \multirow{2}{*}{$\mathbf{T}_{\mathrm{MEF}} / \mathrm{T}_{\mathrm{PVA}}$} & \multirow{2}{*}{$T_{\text {MCA }} / T_{\text {PVA }}$} & \multirow{2}{*}{$T_{\text {MEF }} / T_{\text {MCA }}$} \\
\hline & PVA $^{2}$ & MEF $^{3}$ & MCA $^{4}$ & & & \\
\hline $\mathrm{T}$ & 0.081 & 0.081 & 0.089 & 1.00 & 1.10 & 0.92 \\
\hline L & 0.076 & 0.073 & 0.094 & 0.96 & 1.23 & 0.77 \\
\hline $\mathrm{R}$ & 0.077 & 0.041 & 0.045 & 0.53 & 0.58 & 0.91 \\
\hline
\end{tabular}

Notas:

1. Dirección del modo de vibración: T (Transversal: eje X), L (Longitudinal: eje Y) y R (Rotacional)

2. Prueba de Vibración Ambiental

3. Método de Elementos Finitos

4. Método de la Columna Ancha 
Tabla 2. Periodos de vibración en la vivienda Tipo 2 de concreto normal.

\begin{tabular}{|c|c|c|c|c|c|c|}
\hline \multirow{2}{*}{ Modo } & \multicolumn{3}{|c|}{$\mathrm{T}(\mathrm{s})$} & \multirow{2}{*}{$\mathbf{T}_{\text {MEF }} / \mathbf{T}_{\text {PVA }}$} & \multirow{2}{*}{$\mathbf{T}_{\text {MCA }} / \mathbf{T}_{\mathrm{PVA}}$} & \multirow{2}{*}{$\mathbf{T}_{\text {MEF }} / \mathbf{T}_{\text {MCA }}$} \\
\hline & PVA & MEF & MCA & & & \\
\hline$T$ & 0.065 & 0.066 & 0.083 & 1.02 & 1.26 & 0.80 \\
\hline L & 0.051 & 0.055 & 0.072 & 1.09 & 1.42 & 0.77 \\
\hline R & 0.040 & 0.035 & 0.039 & 0.88 & 0.99 & 0.89 \\
\hline
\end{tabular}

Tabla 3. Periodos de vibración en la vivienda Tipo 2 de concreto celular.

\begin{tabular}{|c|c|c|c|c|c|c|}
\hline \multirow{2}{*}{ Modo } & \multicolumn{3}{|c|}{$\mathbf{T}(\mathbf{s})$} & \multirow{2}{*}{$\mathbf{T}_{\text {MEF }} / \mathbf{T}_{\text {PVA }}$} & \multirow{2}{*}{$\mathbf{T}_{\text {MCA }} / \mathbf{T}_{\text {PVA }}$} & $\mathbf{T}_{\text {MEF }} / \mathbf{T}_{\text {MCA }}$ \\
\cline { 2 - 4 } & PVA & MEF & MCA & 0.94 & 1.17 & 0.81 \\
\hline$T$ & 0.061 & 0.058 & 0.071 & 0.94 & 17 \\
\hline $\mathrm{L}$ & 0.061 & 0.049 & 0.065 & 0.81 & 1.06 & 0.76 \\
\hline $\mathrm{R}$ & 0.041 & 0.032 & 0.036 & 0.77 & 0.86 & 0.89 \\
\hline
\end{tabular}

En cuanto al periodo de vibración fundamental (primer modo) en las dos metodologías numéricas utilizadas, la relación entre el periodo analítico y experimental (TMEF/TPVA y TMCA/TPVA), es adecuada. Sin embargo, los periodos de vibración obtenidos por medio del MEF son, en general, mucho más cercanos a los de las PVA. Adicionalmente, los periodos de los modelos del MCA son mayores que los del MEF, aproximadamente entre $9(1 / 0.92)$ y $25 \%(1 / 0.80)$ para el periodo fundamental de vibración. Esta última tendencia se esperaba, ya que por medio del MCA no se alcanza a modelar adecuadamente las condiciones de frontera de los muros, especialmente los de mayor longitud. Por lo tanto, teniendo en cuenta la gran cantidad de incertidumbres que existen en la elaboración de modelos numéricos de estructuras, se puede inferir que estas metodologías son aceptables para la modelación de viviendas de muros de concreto. Sin embargo, es importante hacer algunas aclaraciones y comentarios:

- Durante la visita de campo se observó el mal estado de la junta de construcción entre las viviendas Tipo 1 de un nivel. Aunque en los modelos se incluyó el efecto de la junta por medio de elementos tipo resorte con rigideces equivalentes aproximadas, es probable que la irregularidad de las propiedades de la junta a lo largo de su longitud altere las frecuencias de vibración obtenidas con las metodologías numéricas, especialmente la frecuencia de vibración en la dirección transversal.

- Como se mencionó anteriormente, muchos de los sistemas de piso que se utilizan en la construcción de vivienda deben catalogarse como diafragmas flexibles. Tal como lo indica Tena (2007), esta es una condición que debe revisarse, pues la respuesta ante cargas laterales entre una estructura con diafragmas rígidos y otra con diafragmas flexibles puede ser muy diferente. En este estudio no se realizó un análisis detallado de los sistemas de piso y se supuso la condición de diafragma rígido. Sin embargo, para el sistema estructural y los claros de estas viviendas, se considera una suposición apropiada. 
- $\quad$ Además de las incertidumbres asociadas con las características propias de las edificaciones analizadas, los resultados obtenidos también dependen del tipo de excitación que se utilice para determinar las características dinámicas. Lo anterior, ya que la estructura puede experimentar diferentes niveles de esfuerzo y no linealidad de los materiales. La determinación de dichas características a partir de PVA, proporciona información del comportamiento estructural asociado a muy bajas amplitudes de excitación y pueden sufrir variaciones significativas durante sismos intensos debido al comportamiento no lineal y al deterioro de la rigidez de los sistemas estructurales (Muriá y González, 1995). Sin embargo, las propiedades dinámicas determinadas a partir de pruebas de pequeña amplitud son un punto de referencia importante para cálculos más detallados y completos (Trifunac, 1972).

VIVIENDAS DE DOS NIVELES. Los resultados de la pruebas de vibración ambiental de la vivienda Tipo 1 de un nivel, también fueron utilizados para establecer los parámetros de calibración en los modelos numéricos de las viviendas de dos niveles. En la Tabla 4 se muestran los periodos de vibración de la vivienda. De forma similar a los modelos de las viviendas de un nivel, los periodos de vibración obtenidos con el MCA son mayores que los del MEF. Cuando se compararon los resultados analíticos con los experimentales en las viviendas de un nivel, se encontró que los periodos de vibración obtenidos con el MEF son mucho más cercanos a los de las PVA. Por lo tanto, para este tipo de viviendas en particular se considera representativo un periodo fundamental de aproximadamente $0.12 \mathrm{~s}(\approx 8 \mathrm{~Hz})$.

Tabla 4. Periodos de vibración en las viviendas de dos niveles.

\begin{tabular}{|c|c|c|c|}
\hline \multirow{2}{*}{ Modo } & \multicolumn{2}{|c|}{ T (s) } & \multirow{2}{*}{ T $_{\text {MEF }} / \mathbf{T}_{\text {MCA }}$} \\
\cline { 2 - 3 } & MEF & MCA & 0.80 \\
\hline $\mathrm{L}$ & 0.121 & 0.152 & 0.93 \\
$\mathrm{~T}$ & 0.110 & 0.119 & 0.93 \\
$\mathrm{R}$ & 0.062 & 0.066 & 0.93 \\
\hline
\end{tabular}

Teniendo en cuenta las consideraciones realizadas anteriormente, es importante mencionar que los resultados encontrados son un indicio del periodo de vibración de este tipo de viviendas y no deben utilizarse para un diseño definitivo de una estructura en particular. Lo anterior, ya que aunque las construcciones tienen sistemas estructurales similares, la variación de alguna o algunas características como su geometría, niveles de esfuerzo, deterioro, características de los materiales, entre otras; pueden originar una variación importante entre las propiedades dinámicas de edificaciones aparentemente similares.

\section{CONCLUSIONES}

A partir de los resultados obtenidos y teniendo en cuenta las incertidumbres en la elaboración de modelos numéricos, se puede concluir que las dos metodologías numéricas 
estudiadas son aceptables para la modelación de viviendas de muros de concreto de baja altura. Si bien es relativamente sencillo elaborar modelos por medio del MCA, las simplificaciones en la concentración de las propiedades del muro en un elemento tipo "barra" y en el apoyo de estos elementos en un solo punto, originan que los resultados obtenidos sean mayores que los de las PVA. Aunque en la modelación por medio del MEF se tienen en cuenta de una manera más detallada las características geométricas y la condición de apoyo de los muros, también se presentan pequeñas diferencias respecto a las PVA. No obstante, los periodos de vibración obtenidos a partir pruebas de vibración ambiental están asociados a amplitudes de excitación bajas y, por lo tanto, es probable que aumenten durante una excitación sísmica.

\section{AGRADECIMIENTOS}

El autor desea expresar su agradecimiento al Dr. Sergio Alcocer Martínez de Castro, profesor investigador del Posgrado en Ingeniería de la Universidad Nacional Autónoma de México (UNAM), por su asesoría durante el desarrollo de la investigación. Así mismo, al Grupo CEMEX (México) por el patrocinio económico de la investigación y al Grupo de trabajo de la Coordinación de Estructuras y Materiales del Instituto de Ingeniería de la UNAM, es especial al Dr. David Muriá Vila y al M.I. Gerardo Rodríguez, por su colaboración durante la realización de las pruebas de vibración ambiental en las viviendas.

\section{REFERENCIAS BIBLIOGRÁFICAS}

[1] BAZÁN, Enrique y MELI, Roberto (2004). Diseño sísmico de edificios. Editorial Limusa S. A., México.

[2] ARISTIZABAL-OCHOA, Dario. (1983). Cracking and shear effects on structural walls. En: Journal of Structural Engineering - ASCE, Vol. 109, No. 5, pp. 1267-1277.

[3] BAZÁN, Enrique y MELI, Roberto (1992). Manual de diseño sísmico de edificios. Editorial Limusa S. A., México.

[4] CARRILLO, Julián (2009). Evaluación del comportamiento a cortante de muros de concreto para vivienda por medio de ensayes dinámicos. Tesis de Doctorado (en proceso). Universidad Nacional Autónoma de México.

[5] CARRILLO, Julián y ALCOCER, Sergio (2008). Shaking table test of low-rise concrete waIls for housing. 14th World Conference on Earthquake Engineering, Beijing, China, Artículo 12-01-0011.

[6] CARRILLO, Julián y ALCOCER, Sergio (2008a). Ensayes dinámicos de muros de concreto con relación de aspecto igual a uno. XVI Congreso Nacional de Ingeniería Estructural, Veracruz, México. Tema VI, Art. 9. 
[7] CHOPRA, Anil y GOEL, Rakesh (2000). Building period formulas for estimating seismic displacements. En: Journal of Earthquake Spectra, Vol. 16, No. 2, pp. 533-536.

[8] CHUN, Young-Soo; YANG, Ji-Soo; CHANG, Kug-Kwan y LEE, Li-Hyung (2000). Approximate estimations of natural periods for apartment buildings with sehar-wall dominant system. 12th World Conference on Earthquake Engineering, Nueva Zelanda. Artículo 0018.

[9] COMPUTER AND STRUCTURES (2005). Static and dynamic finite element analysis of structures - SAP2000 V10.01. Berkeley, California, USA.

[10] FLORES, Leonardo y ALCOCER, Sergio (2001). Estudio analítico de estructuras de mampostería confinada. Informe Técnico. Coordinación de Investigación - CENAPRED. México.

[11] GOBIERNO DEL DISTRITO FEDERAL (2004). Normas técnicas complementarias sobre criterios y acciones para el diseño estructural de las edificaciones (NTC-Cr). Gaceta Oficial del Distrito Federal. México.

[12] GOBIERNO DEL DISTRITO FEDERAL (2004). Normas técnicas complementarias para diseño y construcción de estructuras de concreto (NTC-C). Gaceta Oficial del Distrito Federal. México.

[13] GOBIERNO DEL DISTRITO FEDERAL (2004). Normas técnicas complementarias para diseño por sismo (NTC-S). Gaceta Oficial del Distrito Federal. México

[14] GOEL, Rakesh y CHOPRA, Anil (1998). Period formulas for concrete shear wall buildings. Journal of Structural Engineering - ASCE, Vol. 124, No. 4, pp. 426-433.

[15] INSTITUTO MEXICANO DEL CEMENTO Y DEL CONCRETO - IMCYC (2007). Ensaye de núcleos de concreto. Informe Técnico No. 266 realizado para CEMEX Concretos. México, pp. 2-5.

[16] MURIÁ, David; FUENTES, Luís y GONZÁLEZ, Ricardo (2000). Incertidumbre en la estimación de las frecuencias naturales de vibración de edificios de la ciudad de México. En: Revista de Información Tecnológica, Vol. 11, No. 3, pp. 177-184. México.

[17] MURIÁ, David (1997). Edificios instrumentados. En: El macrosismo de Manzanillo del 9 de Octubre de 1995. Editor: A. Tena, Sociedad Mexicana de Ingeniería Sísmica, Universidad de Colima y Gobierno del Estado de Colima, pp. 197-232.

[18] MURIÁ, David y GONZÁLEZ, Ricardo (1995). Propiedades dinámicas de edificios de la ciudad de México. En: Revista de Ingeniería Sísmica, No. 51, pp. 25-45. México.

[19] TENA, Arturo (2007). Análisis de estructuras con métodos matriciales. Editorial Limusa S. A., México.

[20] TRIFUNAC, Mihailo (1972). Comparisons between ambient and forced vibration experiments. En: Journal of Earthquake Engineering and Structural Dynamics. Vol. 1. pp. 133-150. 